\title{
The Importance of Early Detection of Genetic Diseases
}

\author{
Suma Elcy Varghese ${ }^{a}$ Rana Hassan Mohammad El Otol ${ }^{a}$

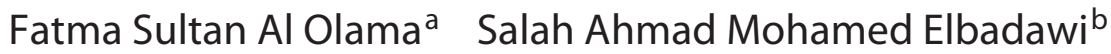 \\ aHealth Affairs Department, Primary Health Care Services Sector, Dubai Health Authority, Dubai, \\ United Arab Emirates; brimary Health Care Services Sector, Dubai Health Authority, Dubai, United Arab Emirates
}

\section{Keywords}

Screening $\cdot$ Newborn $\cdot$ Inborn errors of metabolism .

Hemoglobinopathies · Hemoglobinopathy trait · Sickle cell ·

Thalassemia · Congenital adrenal hyperplasia $\cdot$ Congenital

hypothyroidism · G6PD deficiency

\begin{abstract}
Background: Early detection of diseases in newborn may help in early intervention and treatment, which may either cure the disease or improve the outcome of the patient. Dubai's Health Authority has a newborn screening program which includes screening for metabolic and genetic conditions, for hearing and vision, and for congenital heart disease. Objectives: The objectives of this study are to assess the outcome of the newborn genetic screening program, to correlate the association between the outcome of the program and demographic variables and to find out the percentage of the number of infants who were confirmed to have the genetic disease (by confirmatory tests) out of the total infants who had positive screening test results. Methods: During the period of the study from January 2018 to December 2018, a total of 7,027 newborns were tested in Dubai Health Authority facilities by the newborn genetic screening program (known as the "Step One Screening"). Blood samples were collected by heel prick on a collection paper. All samples were transported to PerkinElmer Genom-
\end{abstract}

(C) 2021 The Author(s)

Published by S. Karger AG, Basel

This article is licensed under the Creative Commons AttributionNonCommercial-NoDerivatives 4.0 International License (CC BYNC-ND) (http://www.karger.com/Services/OpenAccessLicense). Usage and distribution for commercial purposes as well as any distribution of modified material requires written permission. ics in the USA where the tests were done. The genetic disorders identified were correlated with different variables like gender and nationality. The data were entered in an excel sheet and analyzed by using SPSS software. All infants aged 0-3 months who have done newborn genetic screening at Dubai Health Authority facilities between January and December 2018 were included. Results: The incidence of screened disorders was 1:7,027 for congenital adrenal hyperplasia, 1:1,757 for congenital hypothyroidism, 1:1,757 for inborn errors of metabolism, 1:2,342 for biotinidase deficiency, 1:1,171 for hemoglobinopathies, 1:12 for hemoglobinopathy traits, and 1:10 for different genetic mutations of G6PD deficiency. Conclusions: There is a high incidence of different genetic diseases detected by newborn screening. These results justify unifying the program in the UAE and preventive programs like premarital screening and genetic counseling.

(c) 2021 The Author(s)

Published by S. Karger AG, Basel

\section{Introduction}

Newborn screening includes various tests done within a few days of life to detect any congenital disorders. Early detection of diseases may help in early intervention and treatment, which may either cure the disease or improve the outcome and quality of life of the patient. Newborn 
screening includes screening for metabolic and genetic conditions, for hearing and vision, and for congenital heart disease [1].

Robert Guthrie introduced a major breakthrough newborn screening in the early 1960s by doing a bacterial inhibition assay for early detection of phenylketonuria. In phenylketonuria, the neurological damage caused by phenylalanine in diet is irreversible, and early intervention in the form of dietary changes can prevent damage. The introduction of tandem mass spectrometry in the 1990s was a fundamental change in newborn screening as it facilitated screening of many samples for numerous conditions simultaneously in a cost-effective manner [2].

Newborn screening is not just a laboratory test; the test is only a small part of the screening process, and it includes several health professionals. It starts with history and clinical examination of parents before conception and during pregnancy, parental education, and is followed by sample collection and ends with long-term management of positive cases.

A positive screening test result is not a diagnosis. Diagnosis requires further clinical evaluation and biochemical testing. False positives and false negatives can occur, and all the newborn screening programs aim at minimizing them.

A study by Khneisser et al. [3] estimated a reduction of direct cost of care by $50 \%$ per detected case of inborn errors of metabolism. The estimated difference in the cost of care of a late detected case and a case detected by newborn screening was USD 82,000. However, the direct cost to detect a case of inborn error of metabolism was calculated as USD 10,295. This study also showed that newborn screening may be considered economically beneficial even though these diseases are relatively rare especially in countries where there are high rates of consanguinity. From 1995, when the newborn screening program was started in the UAE, $>2,700$ infants were saved from morbidity and associated mortality through screening until 2019 [4].

There are high rates of consanguinity in the Middle East, and hence genetic disorders are relatively common [5]. According to the Ministry of Health Statistics, congenital disorders are now known as the leading cause of infant mortality in the United Arab Emirates. With the advancement in the health care system, preventive programs for genetic disorders became a priority. In 1994, the Ministry of Health established an advisory committee for the prevention of genetic disorders. In 1995, the Central Department of Maternal and Child Health implemented newborn genetic screening in the UAE through the National Screening Center for Woman and Child Health [6]. Since the implementation of the program from January 1995 to December 2014, a total of 983,436 infants were screened by the program. A total of 150 cases $(0.02 \%)$ of inborn errors of metabolism were identified. Other disorders detected were sickle cell disease $(0.05 \%)$, sickle cell trait $(0.85 \%)$, beta thalassemia $(0.005 \%)$, congenital adrenal hyperplasia $(0.01 \%)$, congenital hypothyroidism $(0.05 \%)$, and biotinidase deficiency $(0.01 \%)$. The affected infants were assessed by consultants and management initiated as soon as the diagnosis was confirmed. Confirmed cases were referred for genetic counseling, prenatal testing, and family planning. The committee decided to train the medical staff to counsel parents and establish more genetic clinics in the UAE. They also proposed policies to implement premarital counseling [6].

The Dubai Health Authority started the newborn screening program in 2003 with screening for congenital hypothyroidism and phenylketonuria. Screening for sickle cell disease and congenital adrenal hyperplasia was included in 2005 and 2007, respectively. Tandem mass spectroscopy was introduced in 2011, and this helped in adding many more disorders to the list to be screened. The newborn screening specimens were collected $48 \mathrm{~h}$ after birth by heel prick and transported to the Maternal and Child Health Center in Abu Dhabi for testing. Any abnormal results were notified within 7 days, and parents were directed to hospitals for further investigation. A total number of 139,751 infants were screened during the period 2003-2017, and some of the disorders identified during this period were congenital hypothyroidism $(0.08 \%)$, phenylketonuria $(0.01 \%)$, congenital adrenal hyperplasia $0.03 \%)$, metabolic disorders $(0.03 \%)$, sickle cell disease $(0.06 \%)$, and $\mathrm{Hb} \mathrm{D}$ disease $(0.04 \%)$. However, these data are from patient records and unpublished.

The Dubai Health Authority introduced the "Step One Newborn Screening" in January 2018. More than 50 diseases are screened. This study aims to assess the outcome of the newborn genetic screening program, to correlate the association between the outcome of the program and demographic variables and to find out the percentage of the number of infants who were confirmed to have the genetic disease (by confirmatory tests) out of the total infants who had positive screening test results.

To the best of our knowledge, this is the first study about the outcome of newborn screening in Dubai, UAE. Knowing about the outcome of newborn screening will help emphasize its importance and will help decide on any improvement of practice needed. As many of these disorders are autosomal recessive, their incidence can de- 
crease with proper preventive measures like premarital screening and genetic counseling.

\section{Objectives}

The aims of the study are to assess the outcome of newborn genetic screening, to correlate the association between the outcome of newborn genetic screening and factors like gender and nationality and to find out the percentage of the number of infants who were confirmed to have the genetic disease (by confirmatory tests) out of the total infants who had positive screening test results.

\section{Methodology}

During the period of the study which is from January 2018 to December 2018, a total number of 7,027 newborns were tested in primary health centers and maternity hospitals in the Dubai Health Authority by the newborn genetic screening program (known as the "Step One Screening"), and all of them were included in the study. Trained staff in Dubai government facilities have done the collection of blood samples by heel prick on a collection paper similar to filter paper. The samples collected in the laboratory of each center were kept at a temperature of $2-8^{\circ} \mathrm{C}$ and transported to the Al Bada Primary Health Center laboratory and from there to the National Reference Laboratory (NRL) in Abu Dhabi. The sample is transported in vaccine carriers and temperature charts maintained regularly. NRL transported the samples to PerkinElmer Genomics in the USA where the tests were done. Various methods used for processing the tests were tandem mass spectroscopy, genetic testing, enzyme assays, and biochemical testing. PerkinElmer Genomics has accreditations from the Clinical Laboratory Improvement Amendments (CLIA), College of American Pathologists (CAP), Commission on Laboratory Accreditation (COLA), Join Commission (JC), and External Proficiency Program by Centers for Disease Control and Prevention (CDC).

More than 50 genetic diseases are included in this screening program, and, for the purpose of this study, we categorized these diseases into 5 groups based on their nature; cystic fibrosis, endocrinopathies (congenital hypothyroidism and congenital adrenal hyperplasia), fatty acid and amino acid and organic acid disorders and biotinidase deficiency, hemoglobinopathies and hemoglobinopathy traits, and G6PD deficiency. The genetic disorders identified were correlated with gender and nationality.

The data were entered in an excel sheet and analyzed by using Excel and SPSS 24 software. All the categorical variables are summarized as counts and percentage and presented as tables and bar charts.

The Importance of Early Detection of Genetic Diseases

\section{Results}

During 2018, out of the 6,767 live births in the Dubai Health Authority, 6,595 (97.45\%) were screened by Step One Screening. A total of 7,027 newborns were screened including 432 (6\%) born in private facilities. 4,410 (63\%) are UAE nationals, and 3,686 (52\%) are males.

\section{Cystic Fibrosis}

Five $(0.071 \%)$ infants screened positive for cystic fibrosis, but none had the disease when a confirmatory test was done.

\section{Endocrinopathies}

Two $(0.029 \%)$ Emirati male infants screened positive for congenital adrenal hyperplasia, and both of them did confirmatory tests, but only one of them $(0.014 \%)$ confirmed to have the disease and is on medication. The other infant had inconclusive results initially. The test was repeated again, and the result was normal. Hence, the incidence of congenital adrenal hyperplasia is 1 in 7,027 $(0.014 \%)$ according to this study.

Three males and 1 female $(0.057 \%)$ screened positive for congenital hypothyroidism. All of them did a confirmatory test and were confirmed to have the disease. Three are non-UAE nationals, and one is a UAE national. This study showed that 1 in 1,757 (0.057\%) newborns screened has congenital hypothyroidism.

\section{Metabolic Disorders}

Three infants $(0.043 \%)$ screened positive for biotinidase deficiency. All of them are UAE nationals. They confirmed to have the disease after DNA analysis. Both females have 2 copies of $\mathrm{D} 444 \mathrm{H}$ mutation. Pathogenic heterozygous variant c.322A $>\mathrm{G}$ p. (Ile108Val) and pathogenic heterozygous variant c.1595C $>\mathrm{T}$ p. (Thr532Met) in the biotinidase deficiency (BTD) gene were identified for the male. The incidence of biotinidase deficiency in this study is 1 in $2,342(0.043 \%)$.

Nine infants screened positive for other metabolic disorders. In this group, 6 infants $(0.085 \%)$ showed abnormal acylcarnitine profile, and 3 showed abnormal amino acid profile. Five infants with abnormal acylcarnitine profile and 2 with abnormal amino acid profile did the confirmatory test.

Among the 5 infants who did the confirmatory test for abnormal acylcarnitine profile, 3 confirmed to have inborn errors of metabolism while 2 of them did not have any disease detectable by tandem mass spectroscopy. Among the 2 infants who did the confirmatory test for abnormal amino 


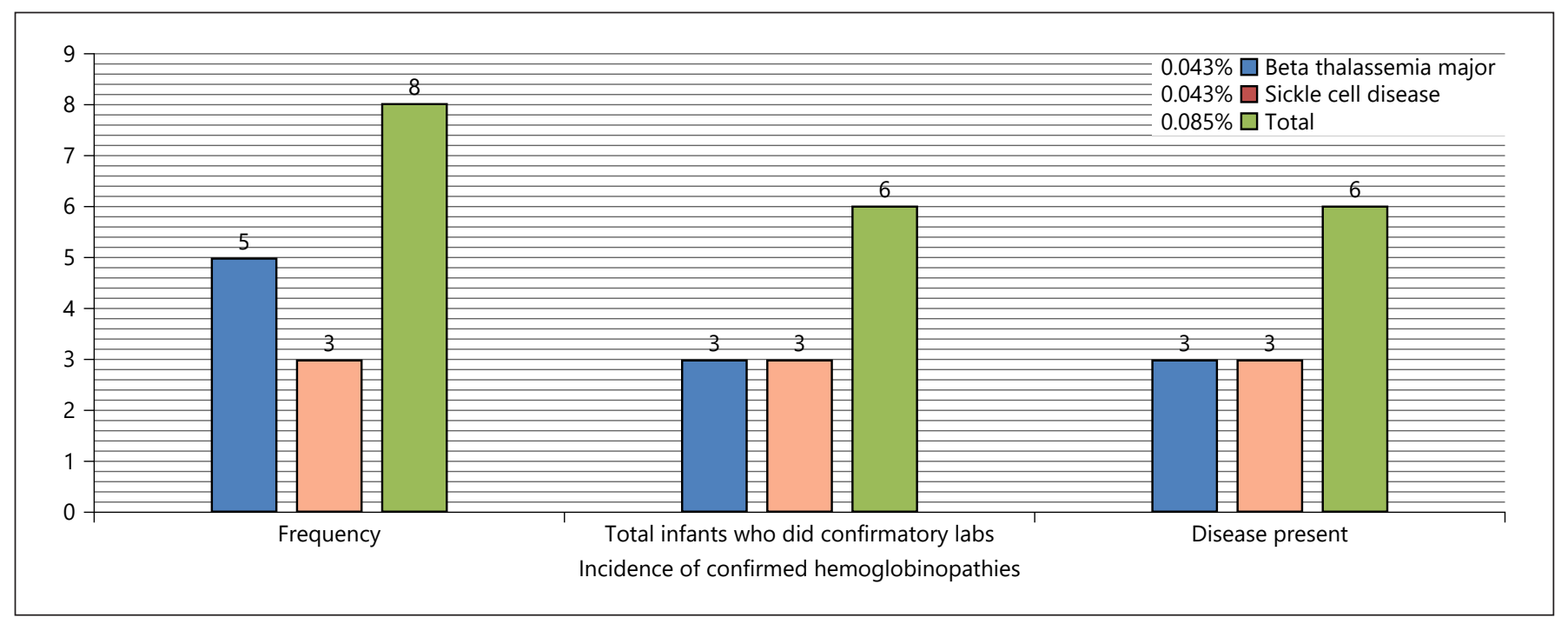

Fig. 1. Counts and percentage of infants screened positive for hemoglobinopathies.

acid profile, one of them confirmed to have mitochondrial DNA depletion syndrome 3 due to DGUOK gene mutation, and the other infant was found to be normal. Both of them were born out of nonconsanguineous marriage. Other inborn errors of metabolism detected were glutaric acidemia type 1, long-chain hydroxyacyl-CoA dehydrogenase deficiency, and multiple Acyl-CoA dehydrogenase deficiency, and these were detected in non-UAE nationals. One of them was born out of second-degree consanguineous marriage, and the other 2 were born out of nonconsanguineous marriage. Incidence of inborn errors of metabolism excluding biotinidase deficiency is 1 in 1,757 .

\section{Hemoglobinopathies}

Eight infants screened positive for hemoglobinopathies. Five of them screened positive for beta thalassemia major and 3 for sickle cell disease (Fig. 1). Out of 5 infants who screened positive for beta thalassemia major, $3 \mathrm{did}$ confirmatory labs, and all were confirmed to have the disease. All infants who screened positive for sickle cell disease were found to have the disease after confirmatory labs (Fig. 1). All infants who were confirmed to have beta thalassemia major and sickle cell disease are Emirati males, and none of them were born out of consanguineous marriage.

\section{Hemoglobinopathy Traits}

611 (8.7\%) infants screened positive for hemoglobinopathy traits. Most common among them is FA+ hemoglobin Barts (trait) which is suggestive of the alpha-thal- assemia trait. Other hemoglobinopathy traits detected were sickle cell, $\mathrm{Hb} \mathrm{D}, \mathrm{Hb} \mathrm{C}, \mathrm{Hb}$ E, and variant $\mathrm{Hb}$. A confirmatory test is routinely not done in case of traits.

FA+ hemoglobin Barts (trait) is the most common hemoglobinopathy trait detected with a frequency of 418 (5.95\%) among the total screened newborns (Fig. 2). The incidence by sex was $6.01 \%$ for males and $5.87 \%$ for females. The incidence by citizenship was 7.73 and $2.94 \%$ for UAE citizens and non-UAE citizens, respectively.

Sickle cell trait showed a frequency of 96 (1.37\%) among the total screened newborns (Fig. 2). The incidence by sex was $1.55 \%$ for males and $1.17 \%$ for females. The incidence by citizenship was 1.52 and $1.11 \%$ for UAE citizens and non-UAE citizens, respectively. Other nonsickle hemoglobinopathies detected showed a lower incidence rate $(\mathrm{Hb} \mathrm{D}-0.77 \%$; variant $\mathrm{Hb}-0.46 \%$; $\mathrm{Hb} \mathrm{E}-$ $0.13 \%$; and $\mathrm{Hb} \mathrm{C}-0.03 \%)$ as shown in Figure 2.

\section{G6PD Deficiency}

Among the 7,027 infants, 709 (10.1\%) screened positive for various genetic mutations of G6PD deficiency. Two types of genetic mutations were identified: C563T mutation and double mutation (G202A; A376G). The most common mutation identified was C563T mutation. Among the 709 infants who screened positive for genetic mutations of G6PD deficiency, 246 (34.7\%) did a confirmatory quantitative G6PD laboratory test. Figure 3 shows the incidence of infants who have the genetic mutation and who were confirmed to have quantitative deficiency of the G6PD enzyme. 


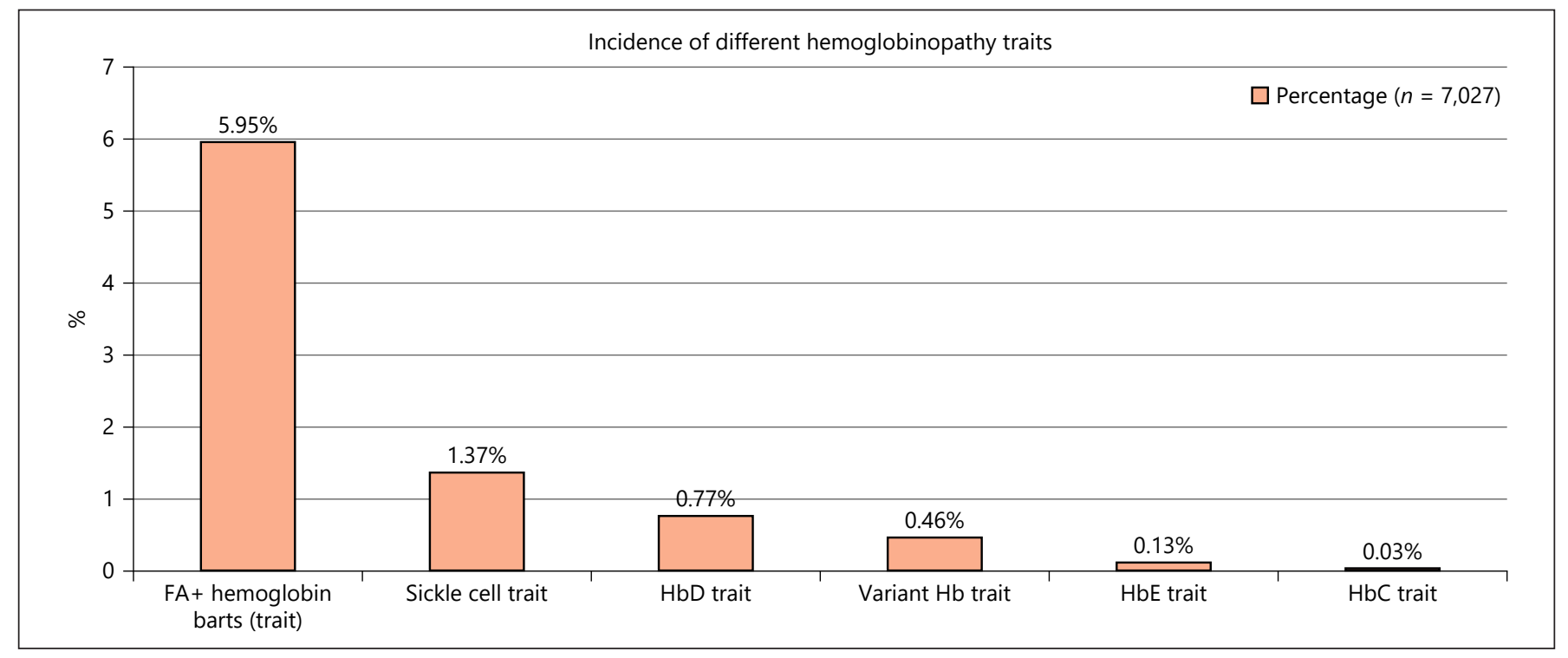

Fig. 2. Incidence of different types of hemoglobinopathy traits detected by step one screening.

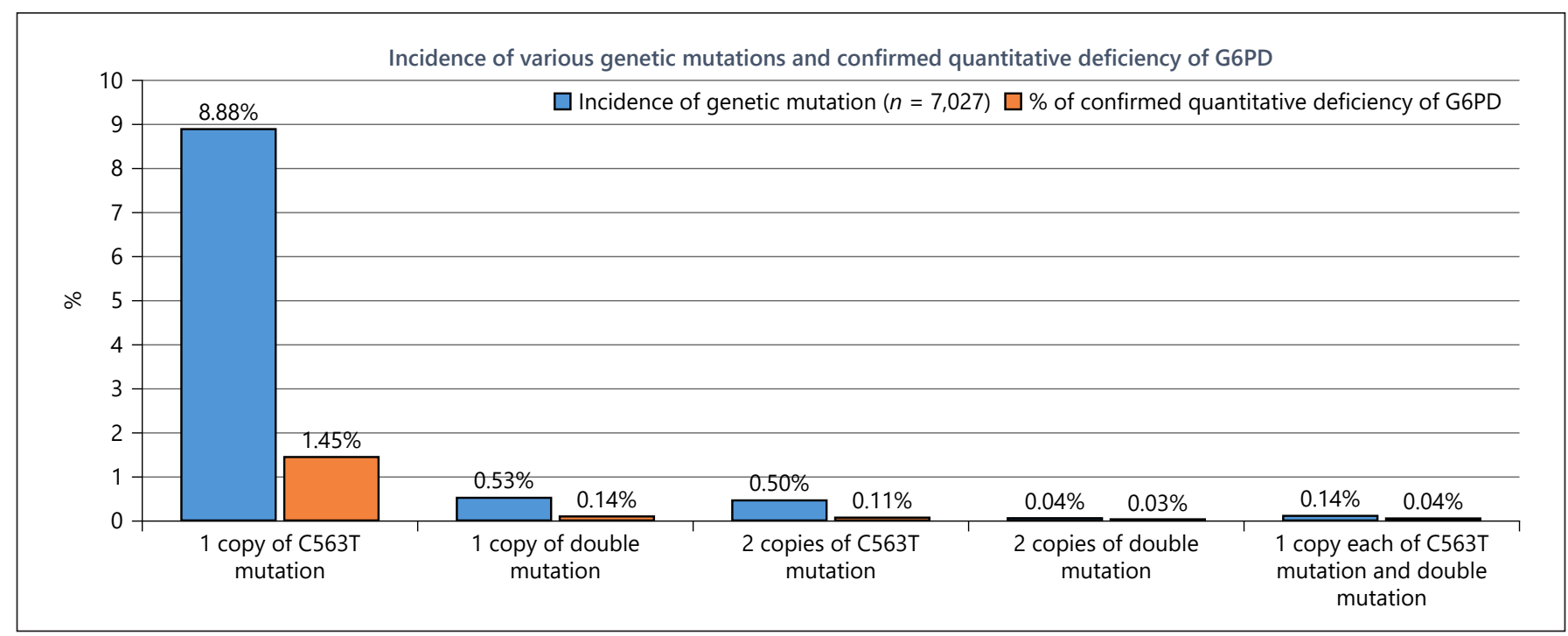

Fig. 3. Incidence of infants who have various genetic mutations and who were confirmed to have quantitative deficiency of the G6PD enzyme.

A total of $624(8.9 \%)$ infants screened positive for G6PD deficiency with 1 copy of C563T mutation. 219 (35\% of the infants who screened positive) among them did a confirmatory test, and $102(1.45 \%)$ confirmed to have a quantitative deficiency of the enzyme. However, all male infants with 1 copy of C563T mutation and who did a confirmatory test had a quantitative enzyme deficiency (Table 1 ) as this disease is an $\mathrm{X}$-linked disorder, and hence males are hemizygous. Thirty-seven $(0.53 \%)$ infants had
1 copy of double mutation (G202A; A376G). Fourteen male infants (37\% of the infants who screened positive) did a confirmatory test, and $10(0.14 \%)$ confirmed to have quantitative deficiency of the G6PD enzyme. Four males with one copy of double mutation (G202A; A376G) had normal G6PD enzyme levels on quantitative testing despite being hemizygous (Table 1). All female infants who did a confirmatory test in the other 3 groups ( 2 copies of C563T mutation, 2 copies of double mutation 


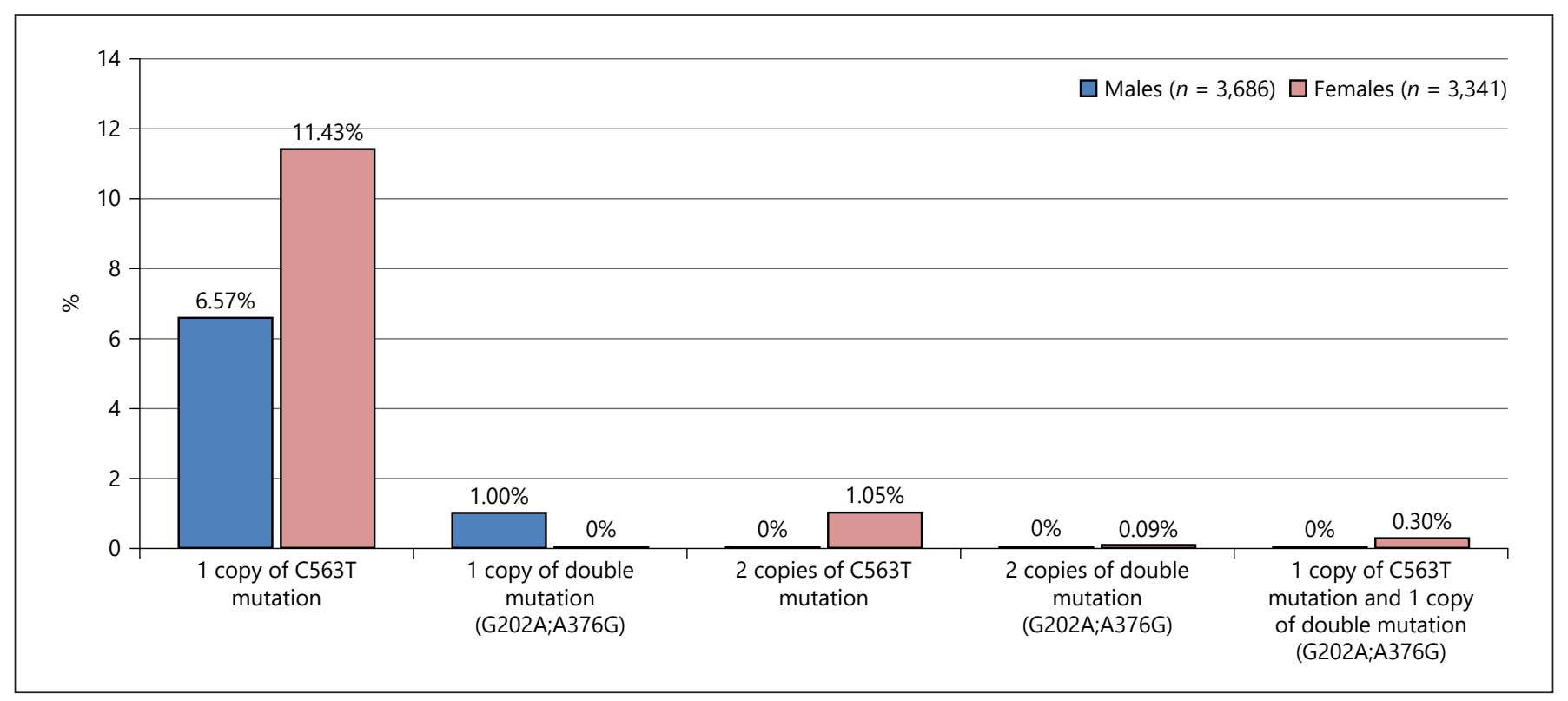

Fig. 4. Incidence of different genetic mutations of G6PD detected by sex.

Table 1. Results of quantitative assay of G6PD in male infant

\begin{tabular}{lllr}
\hline Genetic mutation & $\begin{array}{l}\text { Males with the genetic } \\
\text { mutation (\% of the total of } \\
709 \text { infants who screened } \\
\text { positive), } n(\%)\end{array}$ & $\begin{array}{l}\text { Males with the genetic } \\
\text { mutation and did the } \\
\text { confirmatory labs, } n\end{array}$ & $\begin{array}{l}\text { Males who confirmed to have } \\
\text { quantitative deficiency of } \\
\text { G6PD (\% of those who did } \\
\text { the test), } n(\%)\end{array}$ \\
\hline $\begin{array}{l}\text { One copy of C563T mutation } \\
\text { One copy of double mutation (G202A; A376G) }\end{array}$ & $\begin{array}{c}242 \\
37\end{array}$ & $\begin{array}{r}100 \\
14\end{array}$ & $100(100)$ \\
\hline Total & $279(39.4)$ & 114 & $110(96.5)$
\end{tabular}

[G202A;A376G], 1 copy of C563T mutation, and 1 copy of double mutation [G202A;A376G]) confirmed to have quantitative enzyme deficiency.

Among the 709 infants who screened positive, 430 $(60.6 \%)$ were females and 279 (39.4\%) males. However, as males are hemizygous, more males had quantitative deficiency of the G6PD enzyme when compared with females (Fig. 4). Figures 4 and 5 show the incidence of different types of genetic mutations of G6PD detected, by sex and nationality. Table 1 shows the various types of mutations for G6PD deficiency, frequency of confirmatory test done, and the incidence of confirmed quantitative enzyme deficiency in male infants.

Females who have a single gene for G6PD deficiency may present with low levels of the G6PD enzyme due to compound heterozygosity or lyonization of the normal X chromosome. Step one screening tests for the 5 most common mutations which are responsible for $89 \%$ of cases of G6PD deficiency. Three female infants who had one copy of C563T mutation and all infants with 2 copies of gene mutations had deficient G6PD levels in quantitative assay (Table 2).

\section{Discussion}

Newborn genetic screening was done in 7,027 infants including those born in private facilities, and various genetic disorders were detected. This is the first time that the Dubai Health Authority included screening for cystic fibrosis. There were no confirmed cases of cystic fibrosis during the study period of 1 year. The incidence of cystic fibrosis in the Middle East has been calculated as 1 in 2,000 to 1 in 5,800 live births [7]. 


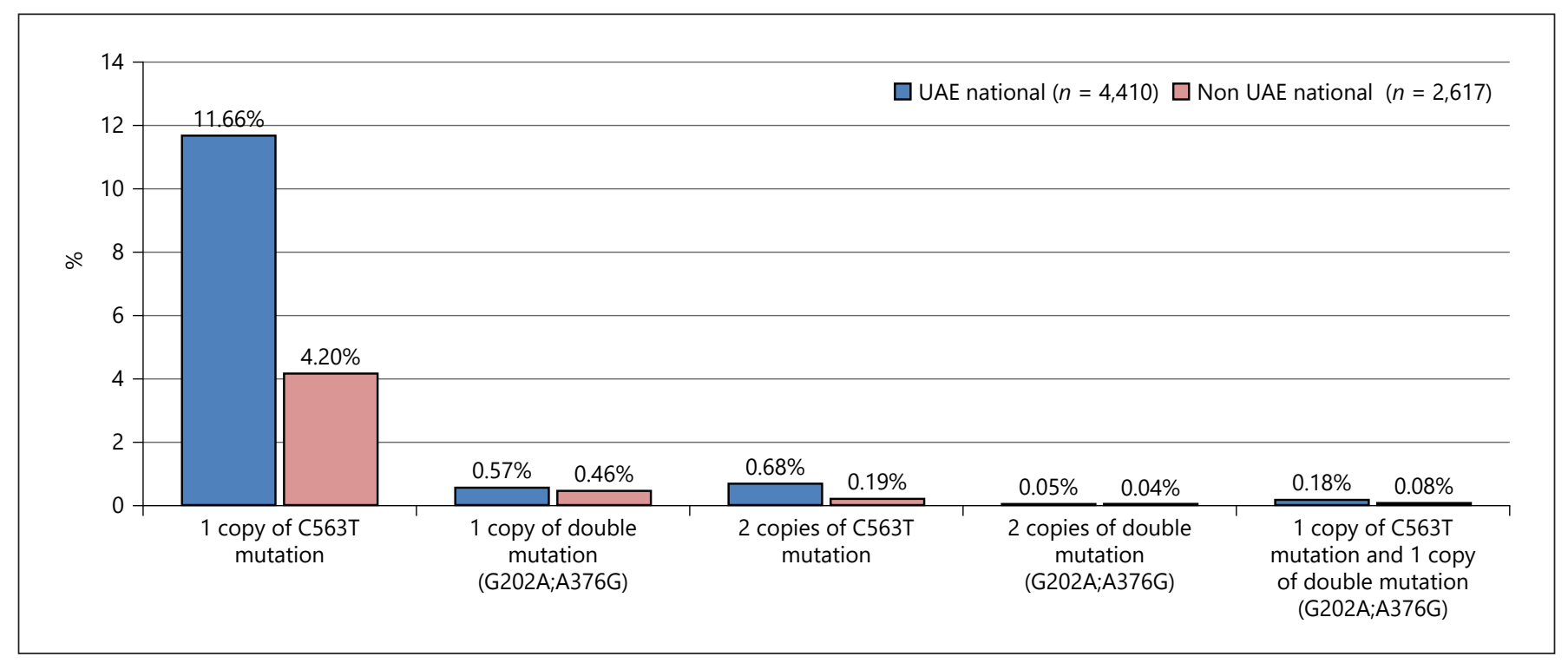

Fig. 5. Incidence of different types of genetic mutations of G6PD detected by nationality.

Table 2. Results of quantitative assay of G6PD in female infants

\begin{tabular}{|c|c|c|c|}
\hline Genetic mutation & $\begin{array}{l}\text { Females with the } \\
\text { genetic mutation } \\
\text { (\% of the total of } \\
709 \text { infants who } \\
\text { screened positive), } \\
n(\%)\end{array}$ & $\begin{array}{l}\text { Females with the } \\
\text { genetic mutation } \\
\text { and did the } \\
\text { confirmatory } \\
\text { labs, } n\end{array}$ & $\begin{array}{l}\text { Females who } \\
\text { confirmed to have } \\
\text { quantitative deficiency } \\
\text { of G6PD (\% of those } \\
\text { who did the test), } \\
n(\%)\end{array}$ \\
\hline One copy of C563T mutation & 382 & 119 & $3(2.5)$ \\
\hline Two copies of C563T mutation & 35 & 7 & $7(100)$ \\
\hline Two copies of double mutation (G202A; A376G) & 3 & 2 & $2(100)$ \\
\hline One copy of double mutation (G202A; A376G) and 1 copy of C563T mutation & 10 & 3 & $3(100)$ \\
\hline Total & $430(60.6)$ & 131 & $110(83.9)$ \\
\hline
\end{tabular}

Incidence of congenital adrenal hyperplasia is 1 in 7,027 according to this study. A similar study by Al Hosani et al. [8] showed an incidence of 1 in 9,030 in the UAE. World-wide incidence of this disorder was estimated at 1:15,000 live births [9]. This study showed that 1 in 1,757 newborns screened has congenital hypothyroidism. A similar study in the UAE from 1995 to 2011 showed an incidence of 1 in 1,873 for congenital hypothyroidism [8]. A study in Qatar showed an incidence of 1 in 3,152 [10], which is lesser when compared with this study. The incidence across the world is described as 1 in 2,000 to 1 in 4,000 after the introduction of newborn screening [11].

Four newborns were confirmed to have different inborn errors of metabolism in this category, and the total incidence was calculated as 1 in 1,757. A similar study in the UAE showed an incidence of 1 in 353 [8]. A more recent study in the UAE showed an incidence of 1 in 2,474 [12] including biotinidase deficiency. Incidence of inborn errors of metabolism was 1 in 1,482 in Lebanon [3]. The incidence of biotinidase deficiency calculated in this study is 1 in 2,342 against an incidence of 1 in 8,300 in a similar study conducted previously in the UAE [8].

Incidence of beta thalassemia major is $0.04 \%$. Incidence of sickle cell disease is also $0.04 \%$, making the total incidence of hemoglobinopathies as $0.08 \%$. Two similar previous studies in the UAE also showed an incidence of $0.04 \%$ for sickle cell disease $[8,12]$. A similar study in Iraq showed an incidence of $0.03 \%$ for beta thalassemia major [13]. 
Sickle cell trait showed an incidence of $1.37 \%$ among the total screened newborns. A similar study in the UAE showed a lower incidence of $0.83 \%$ [8]. The reported prevalence for the sickle cell trait in Saudi Arabia ranges from 2 to $27 \%$ [14].

Alpha-thalassemia carrier frequency can vary among countries and in the UAE, and it was calculated to be $16.4 \%$ in 1986 [15]. The present study shows the incidence as $5.95 \%$ among the total screened neonates. A pilot study in Lebanon showed a frequency of $8 \%$ [16], and another study in Turkey showed 7.5\% [17]. Incidence of nonsickle hemoglobinopathies like $\mathrm{Hb} \mathrm{D}$ and $\mathrm{Hb} \mathrm{C}$ is 0.77 and $0.03 \%$, respectively, in this study. Incidence calculated in a previous study in the UAE was 0.11 and $0.02 \%$ [6].

The present study identified 2 types of mutations responsible for G6PD deficiency. They are C563T and double mutation (G202A; A376G). Five types of mutations were identified in a similar study in China [18], but none of them are similar to the ones detected in the UAE. The prevalence of G6PD deficiency in males and females was 6.0 and $1.5 \%$, respectively, in the same study [18]. Newborn screening in Mexican population revealed an incidence of $0.68 \%$ among males which is much lower compared to $7.58 \%$ in the present study [19]. Single copy of double mutation of G202A:A376G was detected in $97.22 \%$ of cases. However, only $13.26 \%$ male infants have the same double mutation (G202A:A376G) in the present study. $86.73 \%$ males have a single copy of C563T mutation in this study.

\section{Conclusions}

The importance of newborn genetic screening has been emphasized in this study. Infants who screened positive for diseases like the inborn errors of metabolism, endocrinopathies, G6PD deficiency, and hemoglobinopathies were managed early even before they were symptomatic. Therefore, it could prevent or revert severe disabilities and reduce health care costs. It will improve the quality of their lives by reducing complications, hospitalization, and subsequent morbidity and mortality. This emphasizes the importance of newborn screening despite the rarity of these conditions in a country like the UAE where the rates of consanguinity is high. Data about incidence of different diseases in the community will help in planning if any changes need to be made in the newborn screening program. There were infants with positive screening who were lost to follow-up, and in many cases, parents were not reachable over phone, and in other cas- es they were unwilling for further investigations and follow-up. Setting up a dedicated team to follow-up these infants and collecting more contact details might help in this case. The lack of awareness of the seriousness of further investigations and follow-up in the general public should be addressed. Health education by various means like mass media and web pages might help. It is important to unify the newborn genetic screening program throughout the UAE including both the government and private sector. This will help in having precise data on the incidence and prevalence of genetic diseases, unifying the management and follow-up protocols, and help in planning prevention programs. A link between the newborn screening program and the premarital program will help in avoiding repetition of many lab tests, hence reducing health care costs and improving patient satisfaction.

\section{Acknowledgements}

We are overwhelmed in all humbleness and gratefulness to acknowledge all those who helped us to put these ideas well above the level of simplicity. We would like to thank M.Z., F.M., and F.A. who helped us in gathering different information, collecting data, doing the statistical analysis, and guiding us from time to time in making this research.

\section{Statement of Ethics}

Research was done retrospectively, and the source of data was the electronic medical record (Salama). Patient confidentiality and data privacy were maintained. The study protocol was approved by the Dubai Scientific Research Ethics Committee, Dubai Health Authority (Ref: DSREC-08/2019_06, October 08, 2019).

\section{Conflict of Interest Statement}

The authors declare that they do not have any conflicts of interest.

\section{Funding Sources}

The authors did not receive any funding.

\section{Author Contributions}

F.O. provided the data. S.V. and R.O. analyzed it. S.V. wrote the initial manuscript, while R.O. put the data in statistical figures. S.E. edited the final version of the research and gave guidance on necessary changes. All authors reviewed the final manuscript. 


\section{References}

1 Shahied SI, Lubin IM, Jones EA, Tanksley S, Caggana M, Hermerath CA. Newborn screening: from Guthrie to whole genome sequencing. Public Health Rep. 2013;128(5):s14.

2 Millington DS, Kodo N, Norwood DL, Roe CR. Tandem mass spectrometry: a new method for acylcarnitine profiling with potential for neonatal screening for inborn errors of metabolism. J Inherit Metab Dis. 1990;13(3): 321-4.

3 Khneisser I, Adib S, Assaad S, Megarbane A, Karam P. Cost-benefit analysis: newborn screening for inborn errors of metabolism in Lebanon. J Med Screen. 2015;22(4):182-6.

4 Skrinska V, Khneisser I, Schielen P, Loeber G. Introducing and expanding newborn screening in the MENA region. Int J Neonatal Screen. 2020;6(1):12.

5 al-Gazali LI, Bener A, Abdulrazzaq YM, Micallef R, al-Khayat AI, Gaber T. Consanguineous marriages in the United Arab Emirates. J Biosoc Sci. 1997;29(4):491-7.

6 Annual Report 2. National preventive genetic programs. 2014:1.

7 Banjar H, Angyalosi G. The road for survival improvement of cystic fibrosis patients in Arab countries. Int J Pediatr Adolesc Med. 2015;2(2):47-58.
8 Al Hosani H, Salah M, Osman HM, Farag $\mathrm{HM}$, Anvery SM. Incidence of haemoglobinopathies detected through neonatal screening in the United Arab Emirates. East Mediterr Health J. 2005;11(3):300.

9 Merke D, Kabbani M. Congenital adrenal hyperplasia: epidemiology, management and practical drug treatment. Paediatr Drugs. 2001;3(8):599-611.

10 Lindner M, Abdoh G, Fang-Hoffmann J, Shabeck N, Al-Sayrafi M, Al-Janahi M, et al. Implementation of extended neonatal screening and a metabolic unit in the state of Qatar: developing and optimizing strategies in cooperation with the neonatal screening center in Heidelberg. J Inherit Metab Dis. 2007;30(4): 522-9.

11 Multialam H, Simandjuntak RM, Hamid ED. Congenital hypothyroidism. Paediatrica Indonesiana. 2018;34(5--6):170-4.

12 Al-Jasmi FA, Al-Shamsi A, Hertecant JL, AlHamad SM, Souid AK. Inborn errors of metabolism in the United Arab Emirates: disorders detected by newborn screening (20112014). JIMD Rep. 2016;28:127-35.

13 Kadhim KA, Baldawi KH, Lami FH. Prevalence, incidence, trend, and complications of thalassemia in Iraq. Hemoglobin. 2017;41(3): 164-8.

14 Jastaniah W. Epidemiology of sickle cell disease in Saudi Arabia. Ann Saudi Med. 2011; 31(3):289-93
15 White JM, Byrne M, Richards R, Buchanan T, Katsoulis E, Weerasingh K. Red cell genetic abnormalities in peninsular Arabs: sickle haemoglobin, G6PD deficiency, and alpha and beta thalassaemia. J Med Genet. 1986;23(3): 245-51.

16 Farra C, Daher R, Badra R, el Rafei R, Bejjany $\mathrm{R}$, Charafeddine $\mathrm{L}$, et al. Incidence of alphaglobin gene defect in the Lebanese population: a pilot study. Biomed Res Int. 2015;2015: 517679-3.

17 Guvenc B, Yildiz SM, Tekinturhan F, Dincer S, Akyuzluer I, Okten S, et al. Molecular characterization of alpha-Thalassemia in Adana, Turkey: a single center study. Acta Haematol. 2010;123(4):197-200.

18 Tang F, Huang YL, Jiang X, Jia XF, Li B, Feng $\mathrm{Y}$, et al. [Evaluations of newborn screening program performance and enzymatic diagnosis of glucose-6-phosphate dehydrogenase deficiency in Guangzhou]. Zhonghua Er Ke Za Zhi. 2018;56(5):359-63.

19 Cantú-Reyna C, Santos-Guzmán J, CruzCamino H, Vazquez Cantu DL, GómezGutiérrez R, Góngora-Cortéz JJ, et al. Glucose-6-phosphate dehydrogenase deficiency incidence in a Hispanic population. J Neonatal Perinatal Med. 2019;12(2):203-7. 